\title{
A Novel Power Counting Mechanism for Enhanced MBMS Performance in UMTS Networks
}

\author{
Antonios Alexiou ${ }^{1,2}$, Christos Bouras ${ }^{1,2}$ and Evangelos Rekkas ${ }^{1,2}$ \\ ${ }^{1}$ Computer Engineering and Informatics Dept., Univ. of Patras, Greece \\ ${ }^{2}$ Research Academic Computer Technology Institute, 26500 Rio, Patras, Greece \\ alexiua@cti.gr,bouras@cti.gr, rekkas@cti.gr
}

\begin{abstract}
One of the key objectives of Universal Mobile Telecommunications System (UMTS) is the realization of enhanced end-user experience through the provision of rich multimedia services, ensuring in parallel an economical delivery cost for service providers. Multimedia Broadcast/Multicast Services (MBMS) framework of UMTS epitomizes the increasing popularity of such applications by efficiently delivering multimedia traffic to a large number of users and optimizing the radio interface of UMTS networks. For exploiting resource efficiency, MBMS specifications consider the Counting Mechanism which decides whether it is more economic to deliver MBMS multicast traffic over point-to-point (PTP) or point-tomultipoint (PTM) bearers. However, the necessity to further improve MBMS resource efficiency and integrate new technologies such as High Speed Downlink Packet Access (HSDPA) stresses the need for an advanced Counting Mechanism. To this direction, in this paper we propose a novel Power Counting Mechanism which performs optimal power resource utilization and incorporates the HSDPA broadband extension of UMTS for enhanced performance.
\end{abstract}

\section{Introduction}

The major challenge that the mobile telecommunications industry faces today is how to offer a wide range of appealing multimedia services, such as Mobile TV, to mobile users. The expected high penetration of such services translates into optimal resource allocation strategies and improved network performance. A significant step to compensate for these requirements was the introduction of the MBMS framework in the Release 6 UMTS architecture [2], [3]. In MBMS data is transmitted from a single source entity to multiple destinations. 
The main requirement during the provision of MBMS services is to make an efficient overall usage of radio and network resources. Under this prism, a critical aspect of MBMS performance is the selection of the most efficient radio bearer for the transmission of MBMS multicast data. In the frame of switching between different radio bearers, MBMS specifications consider the Counting Mechanism [8] which decides whether it is more efficient to deploy PTP or PTM bearers. Counting Mechanism is an open issue in today's MBMS infrastructure mainly due to its catalytic role in radio resource management (RRM). Current specifications of Counting Mechanism suffer from two major performance inefficiencies. On the one hand Counting Mechanism may lead to significant power wasting, while on the other hand it is characterized by the absence of broadband characteristics.

According to existing Counting Mechanism, the decision on the threshold between PTP and PTM bearers is based on the number of serving MBMS users [8]. However, this criterion for channel type switching may result to significant wasting of the expensive power resources, since it does not take into account the base station's downlink transmission power. Power in UMTS networks is the most limited resource and may lead to significant capacity decrease when misused.

Another inefficiency of the current Counting Mechanism is the absence of key technologies, such as HSDPA. HSDPA introduces a new transport channel, named High Speed-Downlink Shared Channel (HS-DSCH), which optimizes the air interface to support higher data rate services [4], [7]. Although Release '99 transport channels have already been standardized for MBMS transmissions, MBMS over HS-DSCH is an open research topic. More specifically, in PTP mode multiple Dedicated Channels (DCHs) may be used, while in PTM mode a Forward Access Channel (FACH) is configured [8]. However, all the broadband features of HS-DSCH constitute it an ideal candidate for the delivery of MBMS data.

In this paper we propose a novel Power Counting Mechanism that confronts all the above inefficiencies and enhances MBMS performance. Actually, in this paper we further extend and optimize our previous work presented in [9] in order to better utilize power resources and enrich MBMS with broadband characteristics in the frame of $3 \mathrm{G}$ evolution. The proposed Power Counting Mechanism maximizes power efficiency and simultaneously gets advantage of the HSDPA improved performance. We evaluate the proposed mechanism based on a theoretical analysis of downlink power consumption during MBMS multicast transmission. In addition, in order to prove our proposed mechanism's superiority against the current form of Counting Mechanism we present an explicit comparison between the two approaches. Finally, our investigation steps over the conventional macrocellular analysis and focuses on microcellular environments too.

The paper is structured as follows: Section 2 is dedicated to the functional description of the Power Counting Mechanism, while in Section 3 an extended power profile analysis of the three types of radio bearers (DCH, FACH and HS$\mathrm{DSCH}$ ) is provided. Section 4 is devoted to the presentation of the evaluation results. Finally, concluding remarks and planned next steps are briefly described in Section 5. 


\section{Power Counting Mechanism}

Power Counting Mechanism improved performance relies on the exploitation of power resource efficiency and on the integration of HSDPA technology. The proposed mechanism adopts downlink transmission power as the optimum criterion for radio bearer selection. The transport channel with less power consumption is preferred for the delivery of multicast content. Furthermore, in order to enrich MBMS with broadband characteristics, HS-DSCH transport channel is introduced as a multicast bearer in PTP mode. HS-DSCH brings more capacity and enables the mass-market delivery of MBMS services to end users.

Next in this section, we present the architecture and the functionality of the proposed Power Counting Mechanism. The block diagram of the mechanism is illustrated in Fig. 1. The mechanism comprises three distinct operation phases: the parameter retrieval phase, the power level computation phase and the transport channel selection phase. A periodic check is performed at regular time intervals.

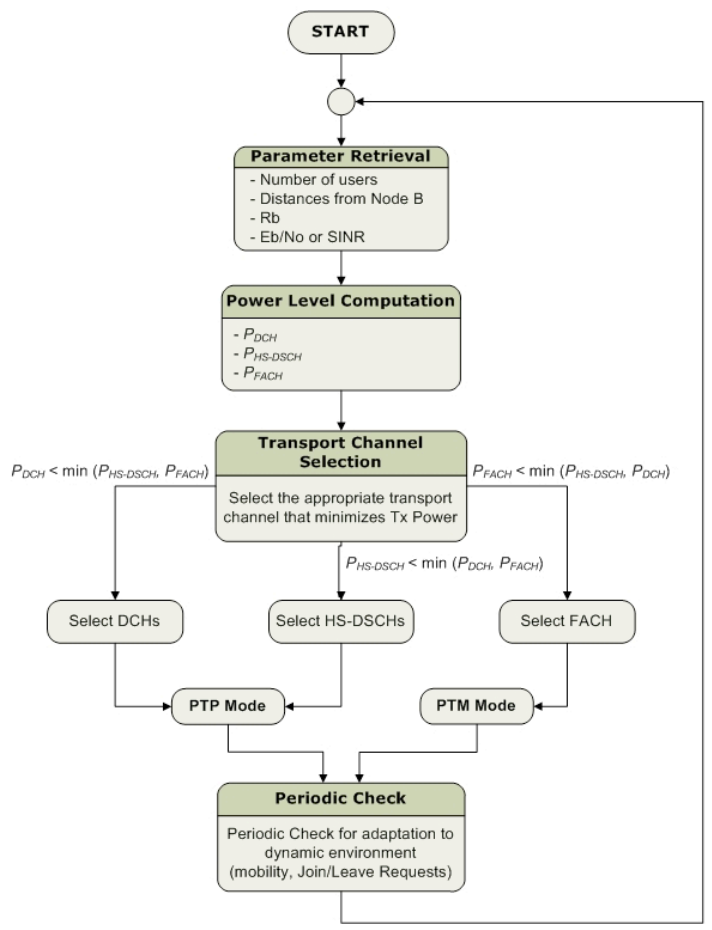

Fig. 1 Power Counting Mechanism

During the parameter retrieval phase (Fig. 1) the mechanism retrieves parameters of the MBMS users and services in each cell. These parameters are the number of users requesting a specific MBMS session, their distances from the base station and their QoS requirements. This information is received from the 
Radio Network Controller (RNC) through appropriate uplink channels. In addition, we assume that the MBMS bit rate service is already known through the Broadcast Multicast-Service Center (BM-SC) node of the MBMS architecture.

The power level computation phase processes the information received from the parameter retrieval phase. In this phase, the required power to be allocated for MBMS session delivery in each cell is computed. The computation is based on the assumption that the transmission of the multicast data can be performed over multiple DCHs, HS-DSCHs or over a single FACH. Consecutively, $P_{D C H}, P_{H S-D S C H}$ and $P_{F A C H}$ power levels are computed respectively for each type of transport channel, according to the method that is presented in Section 3.

In the transport channel selection phase, the appropriate transport channel for the transmission of the MBMS multicast content is selected. The $P_{D C H}$, the $P_{F A C H}$ and the $P_{H S-D S C H}$ are compared. The algorithm decides which case consumes less power and consequently, chooses the corresponding transport channel for the session. This is a key point of our mechanism that actually differentiates Power Counting Mechanism from the existing Counting Mechanism (that uses the number of simultaneous serving users as a selection criterion).

Finally, our mechanism performs a periodic check and re-retrieves user and service parameters in order to adapt to any dynamic changes during the service provision. This periodic check is triggered at a predetermined frequency rate and ensures that the mechanism is able to conceive changes, such as users' mobility, join/leave requests or any fading phenomena and configure its functionality so as to maintain resource efficiency at high levels.

\section{Power Profiles of Downlink Transport Channels}

This section presents the main characteristics of the DCH, FACH and HS-DSCH power profiles. In addition, a theoretical method for the computation of their power consumption levels $\left(P_{D C H}, P_{F A C H}\right.$ and $\left.P_{H S-D S C H}\right)$ during the MBMS multicast transmission is provided.

\subsection{DCH Power Profile}

DCH is a PTP channel and may be used for the delivery of MBMS services to a small number of users. The total downlink transmission power allocated for all MBMS users in a cell that are served by multiple DCHs is variable. It mainly depends on the number of serving users, their distance from the base station, the bit rate of the MBMS session and the experienced signal quality $E_{b} / N_{0}$ for each user. Equation (1) calculates the base station's total DCH transmission power $\left(P_{D C H}\right)$ required for the transmission of the data to $n$ users in a specific cell [5]. 


$$
P_{D C H}=\frac{P_{P}+\sum_{i=1}^{n} \frac{\left(P_{N}+x_{i}\right)}{\frac{W}{\left(E_{b} / N_{0}\right)_{i} R_{b, i}}+p} L_{p, i}}{1-\sum_{i=1}^{n} \frac{p}{\frac{W}{\left(E_{b} / N_{0}\right)_{i} R_{b, i}}+p}}
$$

where $P_{D C H}$ is the base station's transmitted power, $P_{P}$ is the power devoted to common control channels, $L_{p, i}$ is the path loss, $R_{b, i}$ the $i^{\text {th }}$ user transmission rate, $W$ the bandwidth, $P_{N}$ the background noise, $p$ is the orthogonality factor $(p=0$ for perfect orthogonality) and $x_{i}$ is the intercell interference observed by the $i^{\text {th }}$ user given as a function of the transmitted power by the neighboring cells $P_{T j}, j=1, \ldots K$ and the path loss from this user to the $j^{\text {th }}$ cell $L_{i j}$.

\subsection{FACH Power Profile}

FACH is a PTM channel and must be received by all users throughout the MBMS service area of the cell. A FACH essentially transmits at a fixed power level that should be high enough to serve the user with the worst path loss, i.e. the user with the higher distance from the base station. This is another important difference between Power Counting Mechanism and its current form. Existing Counting Mechanism is not scalable and transmits at a power level so as to provide full cell coverage, irrespective of users' location, while Power Counting Mechanism dynamically adjusts its downlink power to a level high enough to serve only the desired cell coverage area.

Table 1. FACH Tx Power Levels vs. Cell Coverage - Macrocell

\begin{tabular}{cc}
\hline \% Cell Coverage & Tx Power $(\mathrm{W})$ - 64 Kbps \\
\hline 10 & 1.4 \\
20 & 1.6 \\
30 & 1.8 \\
40 & 2.0 \\
50 & 2.5 \\
60 & 3.0 \\
70 & 3.6 \\
80 & 4.8 \\
90 & 6.4 \\
100 & 7.6 \\
\hline
\end{tabular}

Table 1 presents some indicative FACH downlink transmission power levels obtained for varying cell coverage areas in a macrocell environment, without 
assuming any diversity techniques. Depending on the distance of the user with the worst path loss from the serving base station, the RNC dynamically sets FACH transmission power at one of the levels presented in Table 1 [6].

\subsection{HS-DSCH Power Profile}

HS-DSCH is a rate controlled rather than a power controlled transport channel. In HSDPA fast power control (characterizing Release '99 channels) is replaced by the Link Adaptation functionality, including techniques such as dynamic Adaptive Modulation and Coding (AMC), multicode operation, fast scheduling, Hybrid ARQ (HARQ) and short Transmission Time Interval (TTI) of $2 \mathrm{~ms}$.

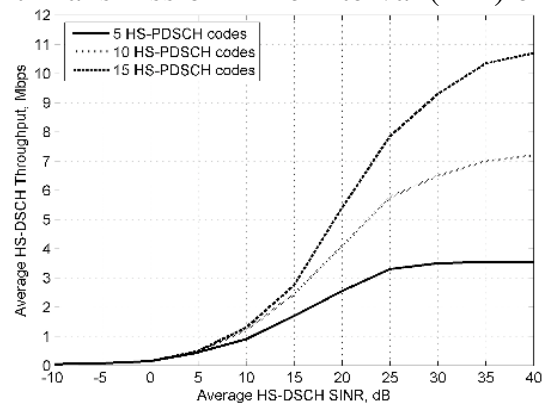

Fig. 2 Actual Cell Throughput vs. SINR

In this paper we employ a dynamic method in order to provide only the required, amount of HS-DSCH power so as to satisfy all the serving users and, in parallel, eliminate system interference. A major measure for HSDPA link budget planning is the HS-DSCH Signal-to-Interference-plus-Noise Ratio (SINR) metric. SINR for a single-antenna Rake receiver is calculated from equation (3):

$$
S I N R=S F_{16} \frac{P_{H S-D S C H}}{p P_{o w n}+P_{o t h e r}+P_{n o i s e}}
$$

where $P_{H S-D S C H}$ is the HS-DSCH transmission power, $P_{\text {own }}$ is the own cell interference experienced by the mobile user, $P_{\text {other }}$ the interference from neighboring cells and $P_{\text {noise }}$ the Additive Gaussian White Noise (AGWN). Parameter $p$ is the orthogonality factor ( $p=0$ for perfect orthogonality), while $S F_{16}$ is the spreading factor of 16 .

There is a strong relationship between the HS-DSCH allocated power and the obtained MBMS cell throughput. This relationship can be disclosed in the three following steps. Initially, we have to define the target MBMS cell throughput. Once the target cell throughput is set, the next step is to define the way that this throughput relates to the SINR (Fig. 2). At this point, it is worth mentioning that 
as the number High Speed-Physical Downlink Shared Channel (HS-PDSCH) codes increases, a lower SINR value is required to obtain a target MBMS data rate (Fig. 2). Finally, we can describe how the required HS-DSCH transmission power $\left(P_{H S-D S C H}\right)$ can be expressed as a function of the SINR value and the user location (in terms of Geometry factor - $G$ ) through equation (4) [7]:

$$
\left.P_{H S-D S C H} \geq S I N K p-G^{-1}\right] \frac{P_{o w n}}{S F_{16}}
$$

The Geometry factor indicates the users' position throughout a cell (distance from the base station). Geometry is given by the relationship between $P_{o w n}, P_{\text {other }}$ and $P_{n o i s e}$ and is defined from equation (5). A lower $G$ is expected when a user is located at the cell edge. Moreover, in microcells MBMS users experience a better (higher) $G$ due to the better environment isolation.

$$
G=\frac{P_{o w n}}{P_{\text {other }}+P_{\text {noise }}}
$$

\section{Performance Evaluation}

For the purpose of the Power Counting Mechanism evaluation we consider the delivery of a typical $64 \mathrm{Kbps}$ MBMS service. Furthermore, both macro and micro cell environments are examined, with parameters presented in Table 2 [1], [10].

Table 2. Macrocell and Microcell Simulation Parameters

\begin{tabular}{lll}
\hline Parameter & Macrocell & Microcell \\
\hline Cellular layout & Hexagonal grid & Manhattan grid \\
Number of cells & 18 & 72 \\
Site-to-site distance & $1 \mathrm{Km}$ & $360 \mathrm{~m}(4$ blocks $)$ \\
Maximum BS Tx power & $20 \mathrm{~W}$ & $2 \mathrm{~W}$ \\
Other BS Tx power & $5 \mathrm{~W}$ & $0.5 \mathrm{~W}$ \\
CPICH Power & $2 \mathrm{~W}$ & $0.2 \mathrm{~W}$ \\
Common channel power & $1 \mathrm{~W}$ & $0.1 \mathrm{~W}$ \\
Propagation model & Okumura Hata & Walfish-Ikegami \\
Multipath channel & Vehicular A $(3 \mathrm{~km} / \mathrm{h})$ & Pedestrian A $(3 \mathrm{Km} / \mathrm{h})$ \\
Orthogonality factor & 0.5 & 0.1 \\
$(0:$ perfect orthogonality $)$ & & $6 \mathrm{~dB}$ \\
$E_{b} / N_{0}$ target & $5 \mathrm{~dB}$ &
\end{tabular}

Initially, we present some indicative results concerning the operation of the transport channel selection phase in order to highlight the key role of power control in MBMS and HS-DSCH's contribution in further enhancing MBMS 
performance. Next, we address the superiority of the proposed mechanism through an explicit comparison, on power and capacity performance, between the Power Counting Mechanism and the existing form of Counting Mechanism.

\subsection{Efficient MBMS Transport Channel Selection}

In this section, we present performance results concerning the most critical aspect of the Power Counting Mechanism: the transport channel selection phase. The mechanism computes the transmission power required for all types of channels and selects the transport channel with less power requirements. This power efficient channel deployment is illustrated in Fig. 3. Transmission power levels for DCH, HS-DSCH and FACH channels are depicted both for macrocell (Fig. 3a) and microcell (Fig. 3b) environments. These power levels, actually, constitute the overall output of the power level computation phase. Users are assumed to be in groups (of varying population each time), located near the cell edge which results to the worst case scenario, in terms of transmission power.

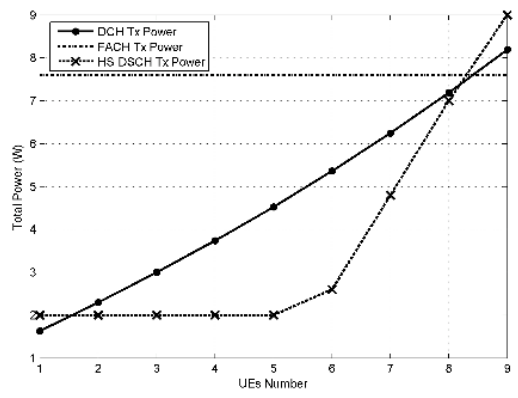

(a)

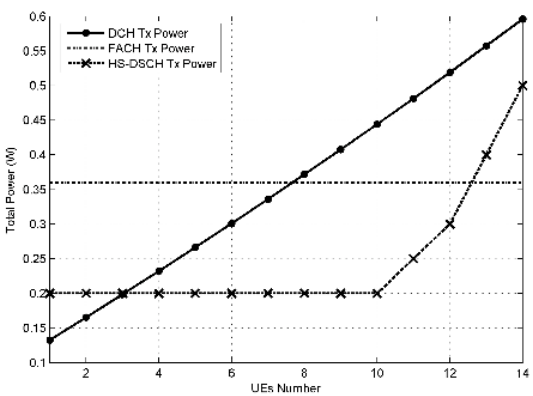

(b)

Fig. 3 MBMS Power Allocation 64Kbps, 100\% coverage (a) Macrocell, (b) Microcell

Regarding the macrocell case we observe, from Fig. 3a, that HS-DSCH is less power consuming for a multicast population with less than 8 users and, thus, it should be preferred for MBMS content transmission (PTP mode). On the other hand, for more than 8 users, FACH is more power efficient and should be deployed (PTM mode). For such a high coverage in macrocells HS-DSCH outperforms the performance of DCH and is the optimal channel in PTP mode. The power gain of HS-DSCH against the DCH reaches $2.5 \mathrm{~W}$ when serving 5 multicast users. Similar results can be extracted in the case of a microcell. However, in microcells it is observed that DCH is the optimal choice for a small multicast group of less than 3 users. For 4-12 users HS-DSCH should be deployed, while for even more users, FACH is the appropriate bearer.

In general, in cases where the number of users is small, PTP transmissions are preferred. DCH and HS-DSCH are both PTP channels; however, the results prove 
that for very small multicast user population $\mathrm{DCH}$ is preferred, while, for relatively more users $\mathrm{HS}-\mathrm{DSCH}$ is the most appropriate. Therefore, our mechanism does not only decide to use PTP or PTM transmissions (as the existing Counting Mechanism does), but it makes a further distinction between DCH and HS-DSCH in PTP mode.

However, the most important notice, extracted from Fig. 3, is that the HSDPA technology provides significant power savings in MBMS PTP mode, when serving a few multicast users, since HS-DSCH appears to be less power consuming than DCH in most cases. This power gain can, in turn, lead to a significant gain in capacity which enables the mass-market delivery of higher bit rate services to end users. As a consequence, it is imperative that HSDPA technology should be integrated in MBMS specifications in order to benefit both operators and mobile users and further improve MBMS resource efficiency.

\subsection{Power Counting Mechanism vs. current Counting Mechanism}

In the previous section, we presented that the Power Counting Mechanism can efficiently utilize power resources. However, the superiority of the mechanism can be better illustrated if we compare the performance of our approach with the current form of the Counting Mechanism. For a more realistic performance comparison, both mobility issues and varying number of serving users are taken into consideration and investigated.

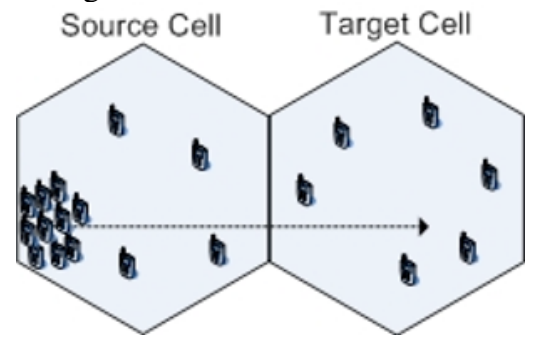

Fig. 4 Simulation Topology

At this point it should be reminded that current Counting Mechanism specifications consider a static switching point between PTP and PTM modes, based on the number of MBMS serving users. Such a reasonable threshold for a macrocell environment would be 8 multicast users. That means that for less than 8 users in PTP mode, multiple DCHs (and no HS-DSCH) would be transmitted, while for more than 8 multicast users in PTM mode, a single $\mathrm{FACH}$, with such power so as to provide full coverage would be deployed.

For the purpose of the evaluation we set up a simulation scenario which considers the provision of a MBMS multicast session in a segment of a UMTS 
macrocellular environment. We examine the performance of both approaches for two neighboring cells (called source cell and target cell) as depicted in Fig. 4. A $64 \mathrm{Kbps}$ MBMS session with $2000 \mathrm{sec}$ time duration is delivered in both cells. Simulation results are depicted in Fig. 5 (source cell) and Fig. 6 (target cell).

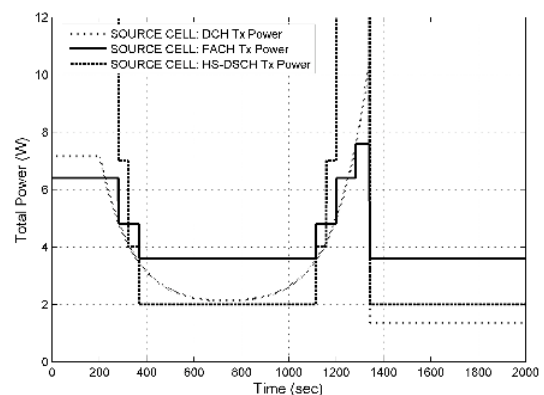

(a)

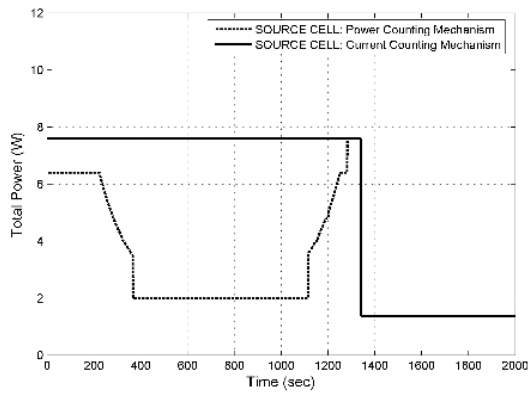

(b)

Fig. 5 Source Cell: (a) Operation of Power Counting Mechanism,

(b) Power Counting Mechanism vs. UE Counting Mechanism

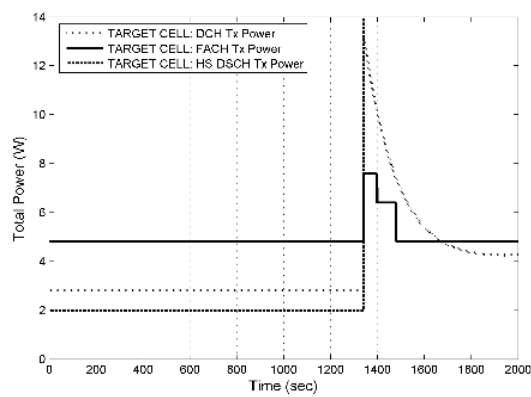

(a)

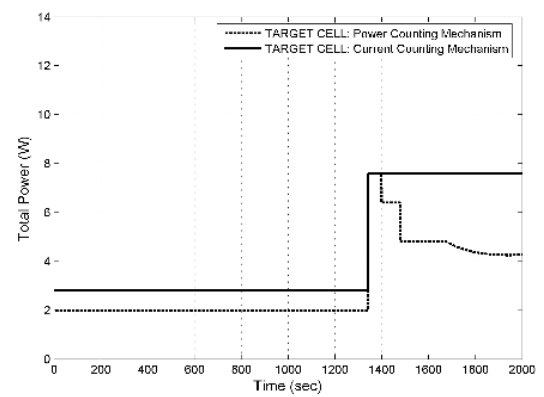

(b)

Fig. 6 Target Cell: (a) Operation of Power Counting Mechanism,

(b) Power Counting Mechanism vs. UE Counting Mechanism

More specifically, Fig. 5a and Fig. 6a depict the downlink power of the three transport channels, as extracted from the power level computation phase, in source and target cells respectively. Fig. 5b and Fig. 6b depict the transmission power of the transport channel that is actually deployed both for the Power Counting Mechanism and the current Counting Mechanism, in source and target cell respectively. In case of Power Counting Mechanism, this power level represents the power consumed by the channel selected in the transport channel selection phase. Regarding the existing Counting Mechanism this power level is either the total DCH power as computed in equation (1) for less than 8 users, or the fixed FACH power, equal to $7.6 \mathrm{~W}$ for full cell coverage, for more than 8 users.

The source cell initially consists of 14 multicast users, while 6 users are residing in the target cell. During the first $200 \mathrm{sec}$ of the simulation time, all users 
in both cells are static. In source cell, the Power Counting Mechanism favors the transmission of MBMS content over FACH with power set to $6.4 \mathrm{~W}$ in order to serve users with the worst path loss, located at a distance of $90 \%$ cell coverage as depicted in Fig. 5a. On the other hand, current Counting Mechanism uses a FACH with $7.6 \mathrm{~W}$ to achieve full cell coverage, since it does not take into account the users' location, resulting in a power wasting of $1.2 \mathrm{~W}$ (Fig. 5b) in the source cell. Target cell is a PTP cell, since it serves less than 8 users. However, HS-DSCH has better performance than DCH, thus, Power Counting Mechanism performs better than the existing Counting Mechanism in the target cell.

A group of 10 users in the source cell, which is located near the cell edge (90\% cell coverage), starts moving at time instance $201 \mathrm{sec}$ towards the target cell, according to the trajectory depicted in Fig. 4, while the rest users remain static. This group leaves the source cell and enters the target cell at time instance 1341 sec. During the time period 201-1341 sec we can make the following observations in the source cell. Power Counting Mechanism is able to track users' location, thus, it dynamically computes power allocation for all transport channels (including the FACH scalable power level). When multicast users get close to the source cell's base station, PTP bearers (DCH and HS-DSCH) are less power consuming than PTM bearer $(\mathrm{FACH})$ even for a large number of serving users. Similarly, when users reside near the cell edge, FACH is more efficient. Existing Counting Mechanism fails to deal efficiently with users' mobility, in the absence of any adaptive procedure, and uses exclusively FACH since simultaneous users receiving the MBMS service exceed the threshold of 8 users. As a result, we observe that a significant power budget (approaching $5.6 \mathrm{~W}$ ) is wasted. Counting Mechanism and Power Counting Mechanism have identical performance only when moving users are on the cell border (a FACH is deployed in both cases).

Finally, at time instance $1341 \mathrm{sec}$, the group of 10 moving users enters the service area of the target cell. At this point, according to current Counting Mechanism, the source cell switches to PTP mode (multiple DCHs) since it serves only 4 users. Power Counting Mechanism also uses DCHs and, thus, both approaches have similar performance. At the same time, the target cell switches to PTM mode (a single FACH) and serves 16 users. However, as the moving group in the target cell keeps moving towards the base station, Power Counting Mechanism appropriately adapts its functionality and leads to better utilization of power resources in contradiction to the static transport channel assignment of the existing MBMS specifications.

Conclusively, from Fig. 5b and Fig. $6 \mathrm{~b}$ it is obvious that the proposed Power Counting Mechanism is prevailing over the current Counting Mechanism. The power based criterion for switching between different transport channels as well as the deployment of the HS-DSCH strongly optimizes resource allocation and enhances MBMS performance. Similar results can also be extracted in the case of microcells. However, in microcells results are even more optimistic due to the fact that HS-DSCH performs better than in macrocells (Fig. 3). In addition, in microcells the higher cell isolation (which entails better geometry distribution) and 
the less multipath propagation ensure the provision of higher MBMS data rate services.

\section{Conclusions and Future Work}

In this paper we proposed a novel Power Counting Mechanism for the efficient transport channel selection in MBMS enabled UMTS networks. The proposed mechanism integrates the HSDPA mobile broadband technology as a part of the overall architecture and defines downlink power as the undisputable switching criterion between different radio bearers. This novel mechanism is capable of conceiving dynamic changes in such wireless environments and optimally adapting its functionality in order to maximize resource efficiency. Simulation results prove that Power Counting Mechanism strongly outperforms current Counting Mechanism of MBMS specifications, by exploiting high power and capacity efficiency.

The step that follows this work is to further examine and optimize the provision of MBMS over HSDPA through experiments conducted in the ns-2 simulator. In addition, power saving techniques that can further improve MBMS performance over HSDPA will be investigated.

\section{References}

1. Holma, H., Toskala, A.: WCDMA for UMTS: HSPA Evolution and LTE. 4th edition, John Wiley \& Sons (2007)

2. 3GPP, TS 22.146 V7.1.0. Technical Specification Group Services and System Aspects; Multimedia Broadcast/Multicast Service; Stage 1 (Release 7)

3. 3GPP, TR 23.846 V6.1.0. Technical Specification Group Services and System Aspects; Multimedia Broadcast/Multicast Service; Architecture and functional description (Release 6)

4. 3GPP, TS 25.308 V5.4.0. Technical Specification Group Radio Access Network; High Speed Downlink Packet Access (HSDPA); Stage 2 (Release 5)

5. Perez-Romero, J., Sallent, O., Agusti, R., Diaz-Guerra, M.: Radio Resource Management Strategies in UMTS. John Wiley \& Sons (2005)

6. 3GPP TS 25.803 V6.0.0, Technical Specification Group Radio Access Network; S-CCPCH performance for MBMS, (Release 6)

7. Holma, H., Toskala, A.: HSDPA/HSUPA for UMTS: High Speed Radio Access for Mobile Communications. John Wiley \& Sons (2006)

8. 3GPP, TS 25.346 V7.2.0. Introduction of the Multimedia Broadcast Multicast Service (MBMS) in the Radio Access Network (RAN), Stage 2, (Release 7)

9. Alexiou, A., Bouras, C., Rekkas, E.: A Power Control Scheme for Efficient Radio Bearer Selection in MBMS. IEEE International Symposium on World of Wireless, Mobile and Multimedia Networks (WoWMoM) (2007)

10.3GPP, TR 101.102 V3.2.0. Universal Mobile Telecommunications System (UMTS); Selection procedures for the choice of radio transmission technologies of the UMTS (UMTS 30.03 version 3.2.0) 